\title{
High dielectric constant poly(vinylidene fluoride-trifluoroethylene- chlorofluoroethylene) for capacitive pressure and bending sensors
}

N. Pereira ${ }^{1,2 \#}$, S. Gonçalves ${ }^{1,2,3 \#}$, J. C. Barbosa ${ }^{1}$, R. Gonçalves ${ }^{4}$, C. R. Tubio ${ }^{5}$, J. L. Vilas-Vilela $^{5,6}$, C. M. Costa ${ }^{1,4,{ }^{*}}$, S. Lanceros-Mendez ${ }^{5,7}$

\#equal contribution

${ }^{1}$ Center of Physics, University of Minho, 4710-058 Braga, Portugal

${ }^{2}$ Centro ALGORITMI, University of Minho, Campus de Azurém, 4800-058 Guimarães, Portugal

${ }^{3}$ EngageLab, University of Minho, 4810-453 Guimarães, Portugal ${ }^{4}$ Center of Chemistry, University of Minho, 4710-058 Braga, Portugal

${ }^{5}$ BCMaterials, Basque Center for Materials, Applications and Nanostructures, UPV/EHU Science Park, 48940 Leioa, Spain.

${ }^{6}$ Departamento de Química Física, Facultad de Ciencia y Tecnología, Universidad del País Vasco (UPV/EHU), Apdo. 644, Bilbao, Spain

${ }^{7}$ Ikerbasque, Basque Foundation for Science, 48009 Bilbao, Spain

*Corresponding authors: C. M. Costa (cmscosta@fisica.uminho.pt)

Keywords: Fluorinated polymers; dielectric constant; capacitive sensors; bending sensors; solvent casting 
Abstract: Poly(vinylidene fluoride-trifluoroethylene-chlorofluoroethylene), P(VDFTrFE-CFE), samples were prepared by solvent casting by varying polymer concentration and solvent evaporation temperature. Different sample morphologies were obtained depending on the processing conditions, as well as different physical-chemical properties such as degree of crystallinity, mechanical and dielectric properties. On the other hand, the electroactive $\beta$-phase content and the degradation temperature of the P(VDF-TrFECFE) samples are independent of the processing conditions. At $1 \mathrm{kHz}$, the highest dielectric constant, $\varepsilon^{\prime}=40$, was obtained for the samples prepared with $15 \mathrm{wt} . \%$ polymer concentration in solutions and processing conditions based on solvent evaporation and polymer melting at $210^{\circ} \mathrm{C}$. Taking into account the high dielectric response, pressure and bending capacitive sensors were developed with excellent response and high sensibility $\left(0.03 \mathrm{kPa}^{-1}\right)$. The developed materials were evaluated for bending and force detection applications. Thus, it is concluded that the developed $\mathrm{P}(\mathrm{VDF}-\mathrm{TrFE}-\mathrm{CFE})$ films are suitable for a new generation of multifunctional materials for capacitive sensing applications, including both force and deformation sensors and touchpads. 


\section{Introduction}

In recent years, smart and multifunctional materials are becoming the base of rapid developments in areas such as sensors and actuators, energy storage and harvesting, wearable electronic devices, and biomedical systems, among others [1-4]. In particular, smart materials are characterized by a controllable, specific and reproducible response to specific stimuli, such as electric and magnetic fields, temperature or pressure [5], being an essential component of the Internet-of-Things [6] and Industry 4.0 paradigms [7].

In particular, piezoelectric smart materials are among the most implemented in sensing applications taking into account their transduction capabilities [8, 9].

Piezoelectric materials convert a mechanical input (applied force) into an electrical output (voltage development) and vice-verse, piezoelectric polymers stand out, when compared to piezoelectric ceramics or single crystals, in terms of flexibility, controllable physical and chemical properties, processability in large areas and a variety of shapes [10] through different processing techniques $[11,12]$, including solvent based ones or from the melt [13].

Electroactive properties of polymers, including electromechanical coupling, dielectric constant or piezoelectric coefficients, are typically lower than in ceramic materials, but more suitable for a large number of applications such as electronic devices, robots, electronic skin, biomedicine, flexible and conformable sensors, among others [14]. Piezoelectric polymers include amorphous and semicrystalline ones, the most investigated and used being poly(vinylidene fluoride), PVDF, due to its high dielectric constant and piezoelectric coefficient compared to other piezoelectric polymers $[15,16]$. PVDF is a semi-crystalline polymer that can crystallize in different phases, $\alpha, \beta, \gamma, \delta, \varepsilon$ that can be obtained depending on the processing conditions, including processing temperature and time or the application or pressure or electric field, among others [17- 
19]. In addition, it can be processed by a variety of techniques, including solvent casting [20], electrospinning [21], extrusion [22] or additive manufacturing techniques, such as printing [23].

Typically, their critical temperatures are $-34{ }^{\circ} \mathrm{C}$ and $160{ }^{\circ} \mathrm{C}$, for the glass transition and the melting temperature, respectively, the melting temperature depending on the crystalline phase. Further, the degree of crystallinity varies between $40 \%$ to $60 \%$ [18]. Its electroactive properties (piezo-, pyro- and ferroelectric) depend on the crystal structure and piezo- and pyroelectric responses are optimized by a poling process used to induce a preferential orientation of the electric dipoles resulting from the difference in the electronegativity between the atoms of carbon and fluorine. The piezoelectric coefficient $d_{33}$ of PVDF in the $\beta$-phase ranges from -18 to $-34 \mathrm{pC} / \mathrm{N}$, depending on the molecular weight, degree of crystallinity and microstructure $[24,25]$. In relation to the dielectric constant, its value is 6 and 9 , for $\alpha$ and $\beta$-phase, respectively, at $25^{\circ} \mathrm{C}$ and $1 \mathrm{kHz}[26,27]$. The $\beta$-phase of PVDF can be obtained under specific processing conditions such as solvent casting at temperatures below $30^{\circ} \mathrm{C}$, by the inclusion of specific fillers such as clays, $\mathrm{BaTiO}_{3}$, ferrites or titanium dioxide, or by high electric field, high temperature and high pressure processing $[10,19]$. Further, copolymers, such as poly(vinylidene fluoridetrifluoroethylene), $\mathrm{P}(\mathrm{VDF}-\mathrm{TrFE})$, have been also developed in order to be able to obtain the polymer in the electroactive phase, independently of the processing conditions [28, 29]. In addition, this copolymer presents the ferro-paraelectric transition (Curie Temperature, $\mathrm{Tc}$ ) below its melting temperature, $\mathrm{T}_{\mathrm{m}}[30]$.

PVDF terpolymers have been also developed, including poly(vinylidene fluoridetrifluoroethylene-chlorofluoroethylene), $\mathrm{P}(\mathrm{VDF}-\mathrm{TrFE}-\mathrm{CFE})$, in which the chlorofluoroethylene (CFE) monomer is introduced in $\mathrm{P}(\mathrm{VDF}-\mathrm{TrFE})$ in order to tune ferroelectricity, improve dielectric constant to values that can reach $\varepsilon^{\prime}=46$, which 
depends on the terpolymer ratio [31] and induce low voltage operation [32]. This terpolymer displays relaxor ferroelectric behavior with a high energy density $\left(0.7 \mathrm{~J} / \mathrm{cm}^{3}\right.$ at $150 \mathrm{MV} / \mathrm{m}$ ) [33], low remnant polarization and breakdown, high thermal stability [34] and glass transition temperature below $-20{ }^{\circ} \mathrm{C}$ [35]. It also presents a distinct electrocaloric behavior [36].

This terpolymer has been implemented in thin-film nonvolatile memories [37], nanoimprinting lithography for polymeric microelectromechanical system (MEMS) devices [38], and in polymer blends based on poly(vinylidene fluoride-co-hexafluoropropylene), $\mathrm{P}(\mathrm{VDF}-\mathrm{HFP})$-another copolymer of PVDF- for energy storage devices [31], as well as in composites with graphene for improving electromechanical response [39].

Taking into consideration the high dielectric constant of this terpolymer in relation to PVDF and its copolymers, its application potential and the scarce literature on the development of $\mathrm{P}(\mathrm{VDF}-\mathrm{TrFE}-\mathrm{CFE})$ films under varying processing conditions to tune their physical-chemical properties, the goal of this work is a systematic study of the processing of $\mathrm{P}(\mathrm{VDF}-\mathrm{TrFE}-\mathrm{CFE})$ by solvent casting, by varying polymer concentration in solution and solvent evaporation temperature. Capacitive pressure and bending sensors have been also developed based on the large dielectric constant of the material. 


\section{Experimental section}

\subsection{Materials}

Poly(vinylidene fluoride-trifluoroethylene-chlorofluoroethylene), P(VDF-TrFE-CFE), (Piezotech® RT-FS; Mw = $500 \mathrm{~kg} / \mathrm{mol} ; 62.5 / 29 / 8.5 \mathrm{~mol} \%$ of VDF, TrFE and CFE, respectively) was provided by Piezotech. N,N-dimethylformamide (DMF, 99.5\%) was supplied by Merck. The materials were used as provided.

\subsection{Sample Preparation}

Two different polymer concentrations in solution were used, $10 \mathrm{wt} . \%$ and $15 \mathrm{wt} . \%$, based on the viscosity of the solutions. Above $15 \mathrm{wt} . \%$, the solution presents a high viscosity of 0.5 Pa.s, whereas below $10 \mathrm{wt} . \%$, the solution is very fluid, not allowing the formation of a consistent films under the selected processing conditions. The samples were prepared by dissolving the polymer powder in DMF under magnetic stirring for 2 hours. After complete polymer dissolution, the solution was spread on a glass substrate by doctor blade with a $50 \mu \mathrm{m}$ spacer. Finally, the solvent was evaporated in an oven (JP selecta) at three different evaporation temperatures. At $210{ }^{\circ} \mathrm{C}$ for 10 minutes, implying also polymer melting; at $90{ }^{\circ} \mathrm{C}$ for 2 hours and at $25^{\circ} \mathrm{C}$ for 15 days. The times were selected to allow complete solvent removal from the sample. The produced films are identified as $x y$, where $\mathrm{x}$ and $\mathrm{y}$ represent the polymer concentration in the solution and temperature, respectively.

\subsection{Sample characterization}

Scanning electron microscopy SEM (SEM, Quanta 650, from FEI) was used to evaluate the surface morphology of the samples, previously coated with a thin gold layer by magnetron sputtering (Polaron SC502 apparatus). Fourier transformed infrared 
spectroscopy (FTIR) was performed in a Jasco FT/IR-6100 set up in the range from 4000 to $600 \mathrm{~cm}^{-1}$ using 64 scans with a resolution of $4 \mathrm{~cm}^{-1}$. The $\beta$-phase $(\mathrm{F}(\beta))$ content of the samples was calculated using equation 1 and the method presented in [19]:

$$
F(\beta)=\frac{A_{\beta}}{\left(\frac{K_{\beta}}{K_{\alpha}}\right) A_{\alpha}+A_{\beta}}
$$

where it is assumed that the polymer crystallizes just in the $\alpha$ or $\beta$ phases, which absorbances (A) correspond to the ones at 766 and $840 \mathrm{~cm}^{-1}$, respectively, and $\mathrm{K}_{\alpha}$ and $\mathrm{K}_{\beta}$ are the absorption coefficients, $6.1 \times 10^{4}$ and $7.7 \times 10^{4} \mathrm{~cm}^{2} \mathrm{~mol}^{-1}$, respectively.

Thermal evaluation was carried out by thermogravimetric analysis (TGA) and Differential scanning calorimetry (DSC). The TGA analysis was performed in a 851e Metter Toledo equipment between $25{ }^{\circ} \mathrm{C}$ and $800{ }^{\circ} \mathrm{C}$, at $10{ }^{\circ} \mathrm{C} / \mathrm{min}$ under nitrogen atmosphere. DSC analysis was achieved in a Mettler Toledo DSC822e equipment in the temperature range from $25^{\circ} \mathrm{C}$ to $200{ }^{\circ} \mathrm{C}$ at $10{ }^{\circ} \mathrm{C} \cdot \mathrm{min}^{-1}$, under nitrogen atmosphere. The degree of crystallinity $\left(\chi_{c}\right)$ of the samples was calculated using equation 2 [19]:

$$
\chi_{c}=\frac{\Delta \mathrm{H}}{\mathrm{x} \Delta \mathrm{H}_{\alpha}+\mathrm{y} \Delta \mathrm{H}_{\beta}}
$$

where $\Delta \mathrm{H}$ is the melting enthalpy of the measured sample and $\Delta \mathrm{H}_{\alpha}$ and $\Delta \mathrm{H}_{\beta}$ are the melting enthalpies of the $\alpha\left(93.07 \mathrm{Jg}^{-1}\right)$ and $\beta$ phase (103.4 $\left.\mathrm{Jg}^{-1}\right)$ of PVDF [40], respectively. The amount of each phase $(\alpha$ and $\beta)$ is represented by $\mathrm{x}$ and $\mathrm{y}$, respectively, which is obtained from the FTIR results. It is to notice that data from pristine PVDF are used for the evaluation of the terpolymer, based on PVDF being the larger part of it and assuming the terpolymer components do not lead to important modifications in the absorption bands and the melting enthalpy, used for the evaluation of the phase content and degree of crystallinity, respectively. 
The mechanical properties of the samples were evaluated at room temperature under tensile stress at a constant deformation of $50 \mu \mathrm{m} \mathrm{s}^{-1}$ with a Linkam Scientific Instruments TST 360 with a load cell of $20 \mathrm{~N}$.

Dielectric measurements were performed using a Quadtech 1920 LCR precision meter. The capacity $(\mathrm{C})$ and the dielectric losses $(\tan \delta)$ were obtained at room temperature in the frequency range from $20 \mathrm{~Hz}$ to $1 \mathrm{MHz}$ with an applied voltage of $0.5 \mathrm{~V}$. Circular aluminum electrodes of $5 \mathrm{~mm}$ diameter were vacuum evaporated onto both sides of each sample with a Polaron SC502 apparatus. The real part of the dielectric function ( $\left.\varepsilon^{\prime}\right)$ was calculated from the data of the measured capacity and the geometrical parameters of the sample, taking into account the parallel plate capacitor geometry. The thickness of the samples was $20 \pm 5 \mu \mathrm{m}$ and the error associated to the dielectric measurements is $\sim 2 \%$. The real part of the a.c. electrical conductivity of the dielectric material was calculated from the dielectric measurements after equation 3:

$$
\sigma^{\prime}(\omega)=\varepsilon_{0} \omega \varepsilon^{\prime \prime}(\omega)
$$

where $\varepsilon_{0}$ is the permittivity of free space, $\omega=2 \pi f$ is the angular frequency and $\varepsilon^{\prime \prime}(\omega)=$ $\varepsilon^{\prime} \tan \delta$ is the frequency dependent imaginary part of the dielectric permittivity [41].

\subsection{Implementation of the capacitive sensor}

The prepared samples were tested as capacity pressure and bending sensors. Unlike some approaches in which pressure is estimated by the variation of the area of contact $[42,43]$, the developed prototype measures the pressure based on the geometrical deformation of the dielectric, which brings the electrodes closer to each other [44]. Therefore, it can be applied as proportional buttons in game controller, scales, dimmable buttons, or bending sensors, among others. 


\subsubsection{Sensor fabrication}

The sensor was built on a polyester (PET) substrate (MELINEX® 506, Dupont, $100 \mu \mathrm{m}$ of thickness), as shown in Figure 1. The bottom circular electrodes, with $20 \mathrm{~mm}$ of diameter, were first screen printed on PET film using silver ink (Novacentrix Metalon ${ }^{\circledR}$ HPS-021LV). The screen is characterized by a mesh size of $264 \times 264$ threads per square inch, with gaps of $55 \mu \mathrm{m}$ and a mesh tension of $20 \mathrm{~N}$. The printed electrodes were cured in an oven (JP Selecta 2005165) for 60 minutes at $80{ }^{\circ} \mathrm{C}$. Then, a $15 \mathrm{wt} . \%$ P(VDF-TrFE-CFE) solution in DMF was spread on a glass substrate and placed in an oven (JP Selecta 2005165) at $210{ }^{\circ} \mathrm{C}$ for 10 minutes for solvent evaporation and polymer melting. After solvent evaporation, all samples are cooled down to room temperature. The resulting $\mathrm{P}(\mathrm{VDF}-\mathrm{TrFE}-\mathrm{CFE})$ films were peeled off from the glass and placed on the PET substrate over the printed electrode and adhered to it by hot pressing (VERSAPRESS FC480) at $100{ }^{\circ} \mathrm{C}$ for $15 \mathrm{~min}$. Then, another silver ink layer was printed by screen printing technique to form the top electrode, and the whole sample was placed in the oven at $80^{\circ} \mathrm{C}$ for 60 minutes for curing and sintering the top electrode and to improve the adhesion strength between P(VDF-TrFE-CFE) and the PET substrate. The thickness of the whole sensor was $127 \mu \mathrm{m}$ : $100 \mu \mathrm{m}$ for the PET film, $20 \mu \mathrm{m}$ for the P(VDF-TrFE-CFE) film and $7 \mu \mathrm{m}$ for the silver electrodes. The active circular sensor layer had a $20 \mathrm{~mm}$ of diameter, determined by the electrodes. 


\section{b)}



Figure 1 - a) Schematic representation of the capacitive sensor layers and the corresponding processing techniques and conditions. Deformation of the sensor in the pressure (b) and bending (c) modes

For the bending sensors, the top electrode was covered by a piece of adhesive tape to protect it from oxidation, whereas for the pressure sensor a polydimethylsiloxane (PDMS), SYLGARD 184 kit from DOW, cover was placed, after being prepared by molding technique using a 3D-printed PLA mold produced by Fused Filament Fabrication (FFF) (Sigma D25, BCN3D Technologies). This elastic cap (Figure 1) gives the user the sensation of pressing an ordinary button, as well as to provide better distribution of forces on the dielectric. Further, the characteristics of PDMS allows simple adhesion to the surface of the sensor, covering the top electrode and preventing it from oxidation. The terminals of the sensor are composed by two traces of silver ink with 20 mm in length, printed along with the electrodes. Then, a z-axis conductive tape (3M 9703) was used to connect the terminals to a $1 \mathrm{~mm}$-pitch flexible flat cable (FFC). 
2.4.2. Principle of operation of the capacitive sensor, electric circuit and data acquisition

The operation of the sensor is based on the variation of the capacitance. When the sensor is pressed or bent, the thickness of the dielectric varies, as represented in Figure 1b) and c), leading to a variation of the overall capacitance, according to:

$$
C=\frac{\varepsilon_{r} \varepsilon_{0} A}{d}
$$

where $C$ (in $\mathrm{F}$ ) is the capacitance, $\varepsilon_{r}$ is the relative permittivity of the dielectric material, $\varepsilon_{0}$ is the absolute permittivity of vacuum $\left(8.854 \times 10^{-12} \mathrm{~F} / \mathrm{m}\right), A$ the area of the electrodes and $d$ the thickness of the dielectric layer. Further, the capacity might also vary due to the change of the overlapping area, particularly in the bending mode, as represented schematically in Figure 1c). Nevertheless, for the deformations under consideration, the contribution of this variation can be neglected.

The instrumentation circuit to read the capacitive sensor consists on a resistor in series with the sensor, as represented in Figure 2a).

a)

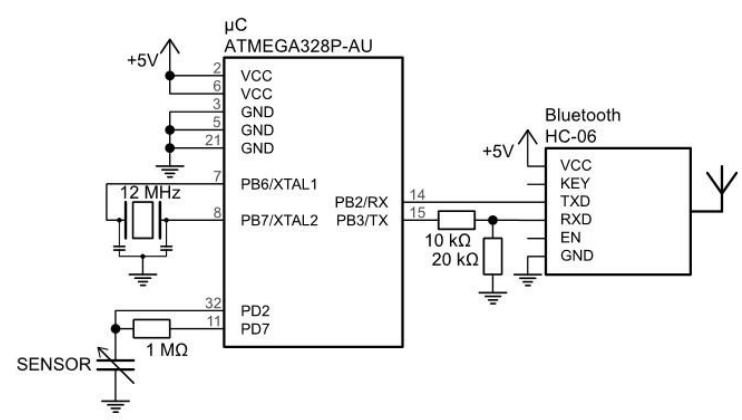

b)

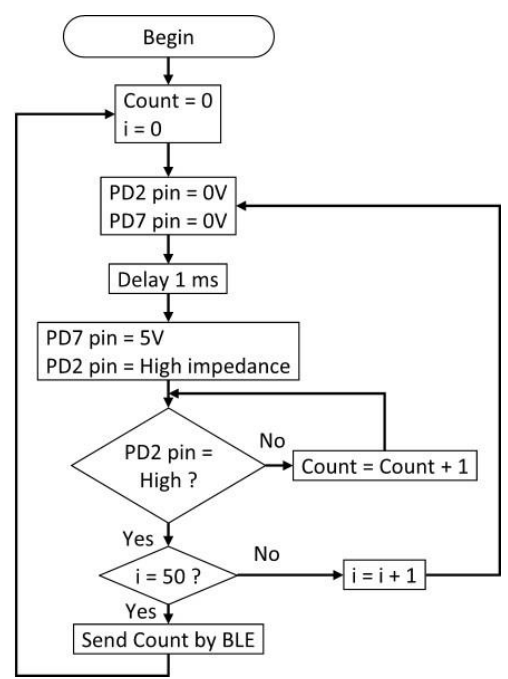

Figure 2 - a) Schematic representation of the readout circuit of the capacity sensor and b) flowchart of the data acquisition algorithm. 
The used microcontroller, $\mu \mathrm{C}$, was a Microchip ATMEGA328P running at $12 \mathrm{MHz}$, connected to a Bluetooth 4.0 (BLE) module (HC-06) via a Universal Asynchronous Receiver/Transmitter (UART) interface. Both the $\mu \mathrm{C}$ and the BLE module were powered at $5 \mathrm{~V}$, but since the transmitting BLE module communication pins supported just a $3.3 \mathrm{~V}$ level, a voltage divider was incorporated between the transmitter pin of the microcontroller (TX) and the receiver pin of the BLE module (RXD). The opposite was not a problem because the $\mu \mathrm{C}$ is tolerant to the $3.3 \mathrm{~V}$ logic level, and therefore the transmitter of the BLE module (TXD) was connected directly to the receiver pin of the $\mu \mathrm{C}(\mathrm{RX})$. The communication baud rate was $9600 \mathrm{bps}$. The data sent via the BLE module were then received by a smartphone where an application developed in Qt 5 displays the plot of the sensor response.

During operation (Figure $2 \mathrm{~b}$ ), the microcontroller $(\mu \mathrm{C})$ measures the time that the sensor capacitor takes to charge. A high value resistor (1 M 2$)$ sets limits to the amount of current that flows into the capacitor. Initially (Figure $2 b$ ), the pin PD2 of the $\mu \mathrm{C}$ is connected to ground level (GND) to fully discharge the sensor. Then, the PD2 pin goes into high impedance input and the PD7 pin is set to $5 \mathrm{~V}$. When the voltage in the sensor reaches a value that the PD2 pin interprets as logic level high, the $\mu \mathrm{C}$ measures the elapsed time since the beginning of the charging of the capacitor. To calculate the time, a counter in polling mode is used, and the returned value, in arbitrary units, changes with the speed of the $\mu \mathrm{C}$, being then converted to capacity in $\mathrm{F}$. In the developed application, data were summed at each 50 events to reduce noise. The full logic of operation is shown in the flowchart of Figure 2b). 


\section{Results and discussion}

3.1. Morphology of the samples

The morphology of the $\mathrm{P}(\mathrm{VDF}-\mathrm{TrFE}-\mathrm{CFE})$ films produced with different polymer concentrations in solution, $10 \mathrm{wt} . \%$ and $15 \mathrm{wt}$, and different processing temperatures, from $25^{\circ} \mathrm{C}$ to $210{ }^{\circ} \mathrm{C}$, is shown in the representative SEM images of Figure 3a to d. For a polymer solution concentration of $15 \mathrm{wt} . \%$, the morphology changes from porous to dense morphology without cracks and defects and with fibrillar texture when the processing temperature increases from $25^{\circ} \mathrm{C}$ to $210^{\circ} \mathrm{C}$. Whereas at temperatures below the melting temperature of the polymer a polymer-solvent phase separation occurs before polymer crystallization, the polymer crystallizing before complete solvent evaporation around the solvent rich regions. Thus, as polymer crystallizes and solvent completely evaporates in this phase separation state, the reduced mobility of polymer chains prevents the polymer to occupy the space left by the solvent during evaporation, leading to the porous structure observed in Figure 3a) [45]. In addition, for a polymer solution concentration of $15 \mathrm{wt} . \%$ and $90^{\circ} \mathrm{C}$ (Figure $3 \mathrm{~b}$ ), the polymer presents a more dense morphology with some voids, indicating that phase separation does not occur completely as verified for $25^{\circ} \mathrm{C}$ as can be also observed in the insert of Figure 3c). Crystallization started in a single phase state and phase separation occurs when small amounts of solvent remain in the sample [46].

At a processing temperature of $210^{\circ} \mathrm{C}$, on the other hand, solvent evaporation occurs right before polymer melting, the polymer crystallizing from the melt as a compact structure (Figure 3c), d)) [47], independently of the initial polymer concentration in solution. This dense morphology with randomly distributed fibrillar texture has been already reported for this polymer when processed from the melt [48]. In fact, it has been demonstrated that these fibrillar textures are related to the crystallites size, high fibrillar textures resulting from high aggregates of crystalline regions and amorphous areas [49]. 

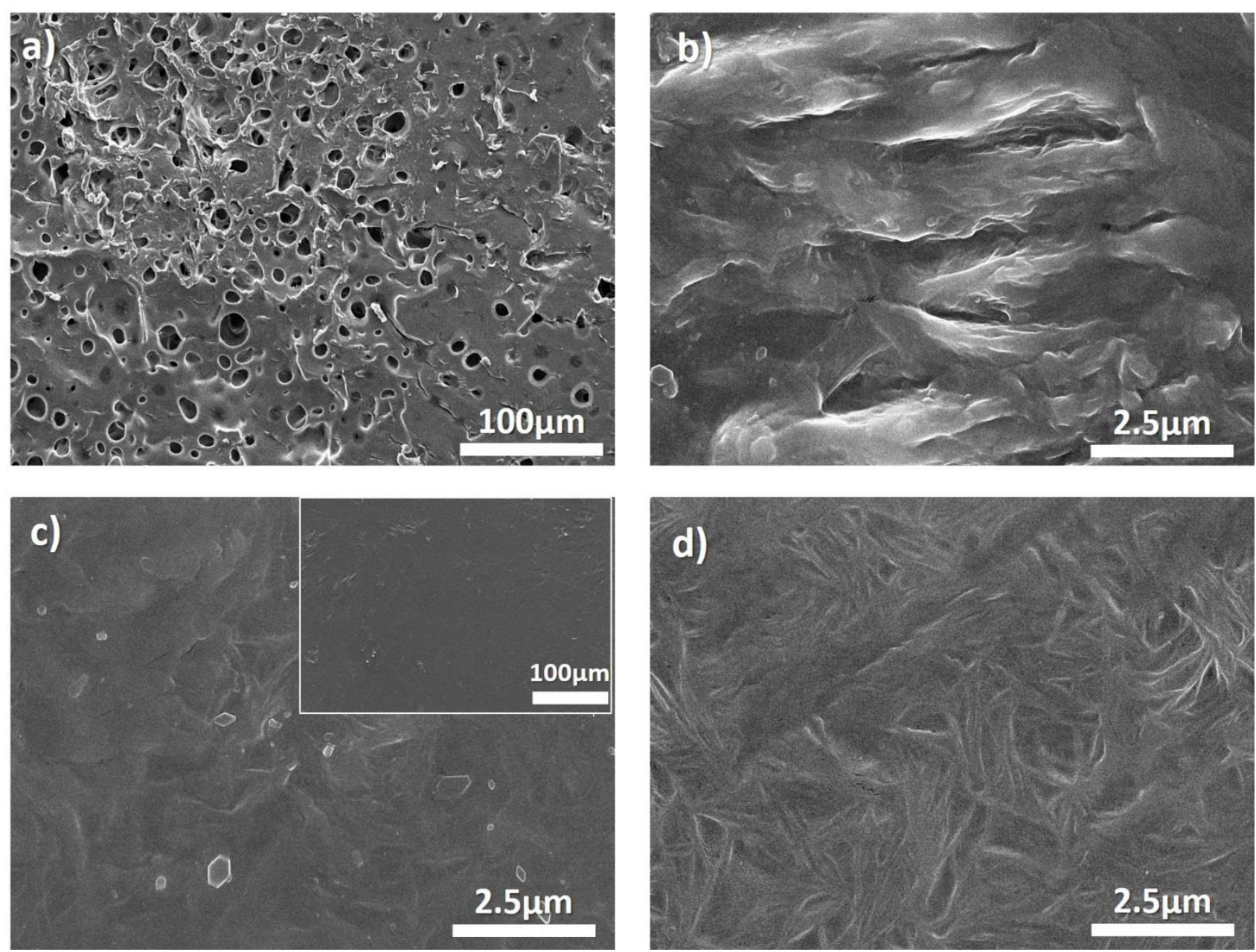

Figure 3 - Representative SEM images of a) 15 wt. $\%-25^{\circ} \mathrm{C}$ sample, b) 15 wt. $\%-90{ }^{\circ} \mathrm{C}$ sample, c) $15 \mathrm{wt} . \%-210{ }^{\circ} \mathrm{C}$ sample and d) $10 \mathrm{wt} . \%-210{ }^{\circ} \mathrm{C}$ sample.

For high temperature processing, $\mathrm{T}=210^{\circ} \mathrm{C}$, decreasing initial polymer concentration in solution from $15 \mathrm{wt} \%$ (Figure 3c) to $10 \mathrm{wt} . \%$ (Figure 3d), increases the fibrillar texture contrast, as low polymer concentrations leads to a slow quenching process and, consequently, increases the number and size of the fibrillar textures. 


\subsection{Polymer phase, thermal and mechanical properties}

The identification and quantification of the crystalline phase of the P(VDF-TrFE-CFE) samples prepared from different polymer concentration in solution and processing temperature was carried out by Fourier transform infrared spectroscopy (FTIR) as presented in Figure 4a). The crystallization in the $\alpha$ - or $\beta$-phases of PVDF depend on the processing conditions and, regardless of the initial polymer concentration and the solvent evaporation temperature up to $80^{\circ} \mathrm{C}$, the nucleation of the $\beta$-phase is promoted due to significant chain entanglements leading to an oriented packing of $\mathrm{CH}_{2}-\mathrm{CF}_{2}$ dipoles [45]. For solvent evaporation temperatures above $120^{\circ} \mathrm{C}$ but below polymer melting, the increase in chain mobility leads to a PVDF crystallization predominantly in $\alpha$-phase transgauche chain conformations due to the non-orientation of the dipolar moments associated with the $\mathrm{CF}_{2}$ bonds [50]. When PVDF is processed from the melt, it crystallizes in the non-polar $\alpha$-phase [10].

In the case of $\mathrm{P}(\mathrm{VDF}-\mathrm{TrFE})$ co-polymer, it crystallizes in the $\beta$-phase, independently of the solvent evaporation temperature or by processing from the melt, for specific $\mathrm{VDF} / \mathrm{TrFE}$ ratios [51], the further inclusion of CTFE units into the copolymer backbone contributing to the destabilization of the polar phase, promoting the formation of the $\alpha$ phase [52].

Regardless of the initial polymer concentration in solution and processing temperature, Figure 4a) shows the typical bands of the P(VDF-TrFE-CFE) polymer at 841 and 1284 $\mathrm{cm}^{-1}$, identifying the ferroelectric $\beta$-phase of the polymer [31]. Further, figure 4a) also shows the characteristic band at $1400 \mathrm{~cm}^{-1}$, related to $\mathrm{CH}_{2}, \mathrm{CF}_{2}$ rocking and $\mathrm{CF}_{2}$ antisymmetric stretching vibration bands [31]. 
The $\beta$-phase content in the samples was quantified after equation 1 and the corresponding values are summarized in Table 1, the electroactive crystalline phase content being $~ 83 \%$ for all $\mathrm{P}(\mathrm{VDF}-\mathrm{TrFE}-\mathrm{CFE})$ samples, independently of the initial polymer concentration in solution and processing temperature.

Therefore, the $\mathrm{P}(\mathrm{VDF}-\mathrm{TrFE}-\mathrm{CFE})$ samples show higher $\beta$-phase content compared to the pristine PVDF polymer processed at temperatures above $90{ }^{\circ} \mathrm{C}[35,53]$, allowing processing in the electroactive phase from solution or melt, as well as compact morphologies suitable for sensors and actuator applications.

Figure 4b) shows the DSC heating thermograms of the different P(VDF-TrFE-CFE) samples. It is observed that, independently of polymer concentration and processing temperature above $90{ }^{\circ} \mathrm{C}$, an endothermic peak is observed around $\sim 130{ }^{\circ} \mathrm{C}$ that correspond to the melting temperature $\left(\mathrm{T}_{\mathrm{m}}\right)$ of the paraelectric crystalline phase [34].

On the other hand, for the samples prepared at solvent evaporation temperatures of $25^{\circ} \mathrm{C}$, figure $4 \mathrm{~b}$ shows two endothermic peaks at $25^{\circ} \mathrm{C}$ and $120^{\circ} \mathrm{C}$ that correspond to the ferroelectric-paraelectric phase transition and the melting temperature, respectively. Crystallization above $50^{\circ} \mathrm{C}$ produces thinner lamellar crystals of the paraelectric phase in the $\mathrm{P}(\mathrm{VDF}-\mathrm{TrFE}-\mathrm{CFE})$ terpolymer, leading to lower melting temperatures [34]. In fact, it has been reported that the addition of the CFE monomer to P(VDF-TrFE) reduces the crystallite sizes and consequently the critical temperatures $\left(\mathrm{T}_{\mathrm{c}}\right.$ and $\left.\mathrm{T}_{\mathrm{m}}\right)$ [35]. Further, the ferroelectric phase transition is not observed in the temperatures processed at temperatures above $50{ }^{\circ} \mathrm{C}$ due to the presence of the ferroelectric crystals as a metastable phase within the crystalline regions of $\mathrm{P}(\mathrm{VDF}-\mathrm{TrFE}-\mathrm{CFE})$, well developed ferroelectric terpolymer crystals being obtained from solution at low temperature rather than from the melt state (Figure 4b). This fact is also verified through annealing treatments, leading to the formation of well-developed FE crystals and, therefore, of the phase transition [35]. 

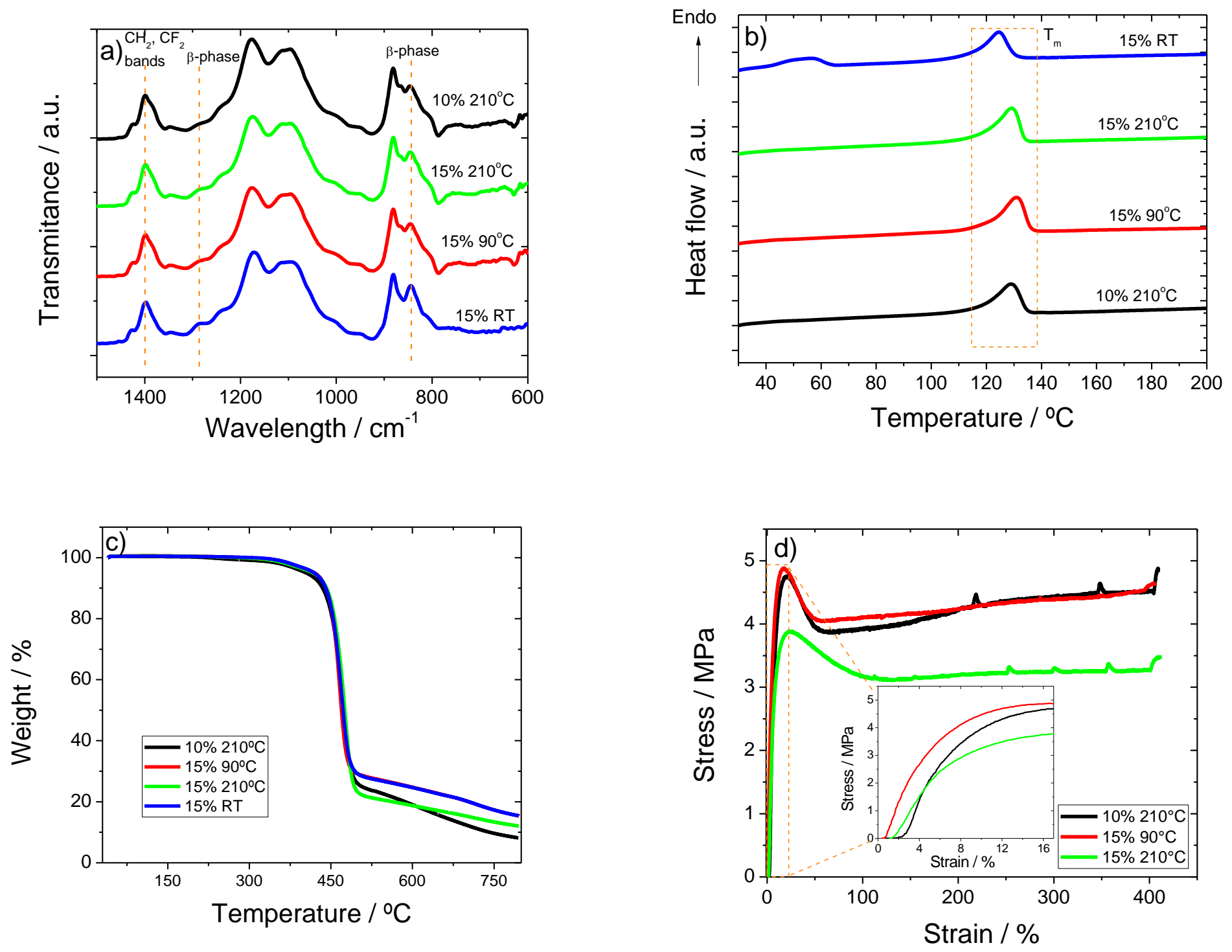

Figure 4 - a) FTIR spectra, b) DSC thermograms, c) TGA curves and d) stress-strain mechanical measurements for the samples prepared under different processing conditions. The inset of figure d) shows the detail of the stress-strain mechanical measurements up to the yielding strain.

The quantification of the degree of crystallinity of the samples was carried out by applying equation 2 to the melting thermograms and the obtained values are summarized in table 1 . The degree of crystallinity is around $\sim 12 \%$ excepting for the sample prepared 
form a polymer concentration of $15 \mathrm{wt} . \%$ and solvent evaporation temperature of $90{ }^{\circ} \mathrm{C}$, which is slightly higher (17\%). This value is slightly lower than that observed in the literature $(\Delta \chi=25 \%)$ since this sample has different CFE content [54] and also for the PVDF-TrFE polymer $(\Delta \chi=26-32 \%)$ [51] in which the addition of the CFE monomer decreases the degree of crystallinity.

Table 1 - Electroactive $\beta$-phase content, degree of crystallinity, Young modulus and yield strength of the samples prepared under different processing conditions.

\begin{tabular}{|c|c|c|c|c|}
\hline Sample & $\boldsymbol{\beta}$-phase $/ \mathbf{\pm 2 \%}$ & $\boldsymbol{\Delta \chi} / \mathbf{\pm 2 \%}$ & $\mathbf{E} / \mathbf{\pm 4 0} \mathbf{~ M P a}$ & Yield Strength / $\mathbf{0 . 5 M P a}$ \\
\hline $15 \%-210^{\circ} \mathrm{C}$ & 83 & 12 & 423 & 2.75 \\
\hline $15 \%-90^{\circ} \mathrm{C}$ & 84 & 17 & 807 & 2.56 \\
\hline $10 \%-210^{\circ} \mathrm{C}$ & 82 & 13 & 780 & 2.51 \\
\hline $15 \%-\mathrm{RT}$ & 86 & 12 & ---- & \\
\hline
\end{tabular}

The thermal degradation of $\mathrm{P}(\mathrm{VDF}-\mathrm{TrFE}-\mathrm{CFE})$ samples, as evaluated from the TGA thermograms presented in Figure 4c), shows that, regardless of processing conditions, a single degradation step is observed around $\sim 466{ }^{\circ} \mathrm{C}$, similar to that observed for the PVDF polymer [55] and P(VDF-TrFE) co-polymer [51]. The degradation temperature is attributed to the scission of carbon - hydrogen $(\mathrm{C}-\mathrm{H})$ bonds, followed by the release of HF molecules, leading to the formation of carbon-carbon double bonds [56].

The mechanical properties (Figure 4d) were just evaluated in the films with dense morphology, as in the porous samples the mechanical properties are determined by the pore size and distribution and not by the polymer itself [57]. All films show the mechanical behavior typical of a thermoplastic polymer, characterized by two main regions: the elastic and plastic regions, separated by the yielding stress/strain [48]. The Young modulus was determined at $3 \%$ of elongation in the elastic region by the tangent 
method and its value for the different samples is presented in table 1, together with the yield strength.

Table 1 shows that the mechanical behavior of the $\mathrm{P}$ (VDF-TrFE-CFE) films is dependent on the initial polymer concentration in solution and the processing temperature, the sample with higher Young modulus being the one prepared from a $10 \mathrm{wt} . \%$ polymer solution and with the solvent evaporated at $90{ }^{\circ} \mathrm{C}$, which is the sample with a higher degree of crystallinity [39]. In relation to the samples processed by melting at $210{ }^{\circ} \mathrm{C}$, Table 1 shows that the Young modulus decreases with decreasing initial polymer concentration due to differences observed in the morphologies as shown in Figure 1.

\subsection{Electrical properties}

The evaluation of the dielectric behavior is essential for capacitive sensing applications. The dielectric behavior has been determined just for the dense film samples, as for the porous sample the dielectric response will be strongly influenced by the free porous space. Figure 5 a) shows the room temperature variation of the dielectric constant, $\varepsilon$, for the different $\mathrm{P}(\mathrm{VDF}-\mathrm{TrFE}-\mathrm{CFE})$ films as a function of frequency.
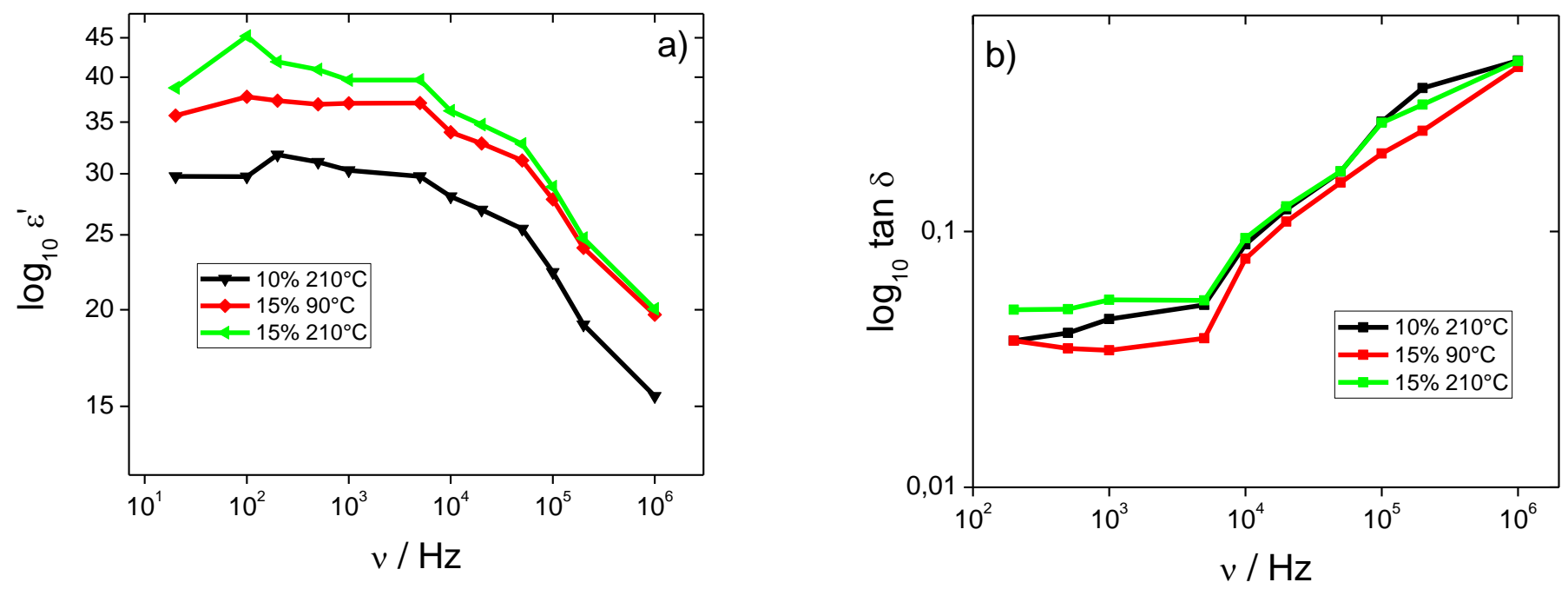

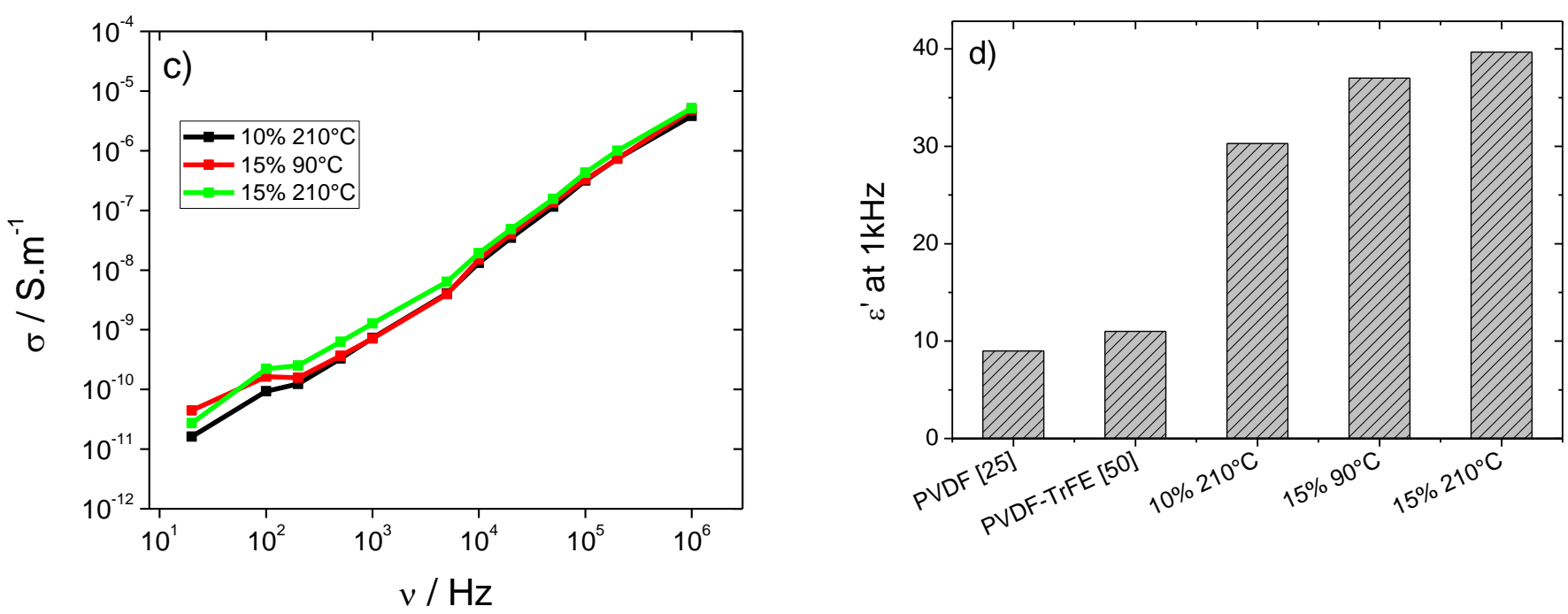

Figure 5 - a) Dielectric constant, b) tangent delta and c) a.c. electrical conductivity of the samples as a function of frequency. d) Dielectric constant of the different samples at a frequency of $1 \mathrm{kHz}$ and comparison with the PVDF polymer [26] and PVDF-TrFE copolymer [51].

Figure 5a) shows that the dielectric constant decreases with increasing frequency for all samples, showing that just one relaxation dominates the terpolymer in this frequency and temperature range [53]. Furthermore, regardless of the processing conditions, figure 5d) shows a high dielectric constant of $\mathrm{P}(\mathrm{VDF}-\mathrm{TrFE}-\mathrm{CFE})$ in relation to PVDF polymer [26] and $\mathrm{P}(\mathrm{VDF}-\mathrm{TrFE})$ copolymer [51] due to its relaxor ferroelectric characteristics in which the inclusion of CFE monomer to the VDF-TrFE chains interrupts the long-chain polarconformation and leads to a rapid change in molecular conformation between a dipolar glass state and randomly interacting polar microregions $[53,58]$. The observed variations in the dielectric constant among the differently processed samples, as represented in the in figure 5d) at $1 \mathrm{kHz}$, is related to the previously described differences in microstructural features and degree of crystallinity of the films [46]. In relation to $\tan \delta$ (figure $5 \mathrm{~b}$ )), it increases with increasing frequency and is related to the movement of crystallineamorphous interphase chain segments [59]. 
The increase of the dielectric losses with increasing frequency, similar for all processed samples, also leads to a corresponding increase of the a.c. electrical conductivity, showing that the losses are related to dipolar and/or confined ionic local movements within the amorphous chain segments (Figure 5c). Finally, the dielectric constant at $1 \mathrm{kHz}$ is presented for the different samples in figure 5d), together with the ones of PVDF polymer and PVDF-TrFE copolymer, showing the advantages of the terpolymer for applications where high dielectric constant is a relevant parameter.

\subsection{Capacitive sensor evaluation}

Taking into account the high dielectric constant of the $\mathrm{P}(\mathrm{VDF}-\mathrm{TrFE}-\mathrm{CFE})$ films prepared from an initial polymer concentration in solution of $15 \mathrm{wt} . \%$ and processed at $210^{\circ} \mathrm{C}$, this sample was used for the development of the sensing elements.

The sensors and the electronic circuit were tested during manual user operation in pressure and bending sensing modes, as presented in Figure 6.

Representative sample sensing response data are plotted in Figure $6 c-d$ for pressure (figure 6c) and bending modes (figure 6d).
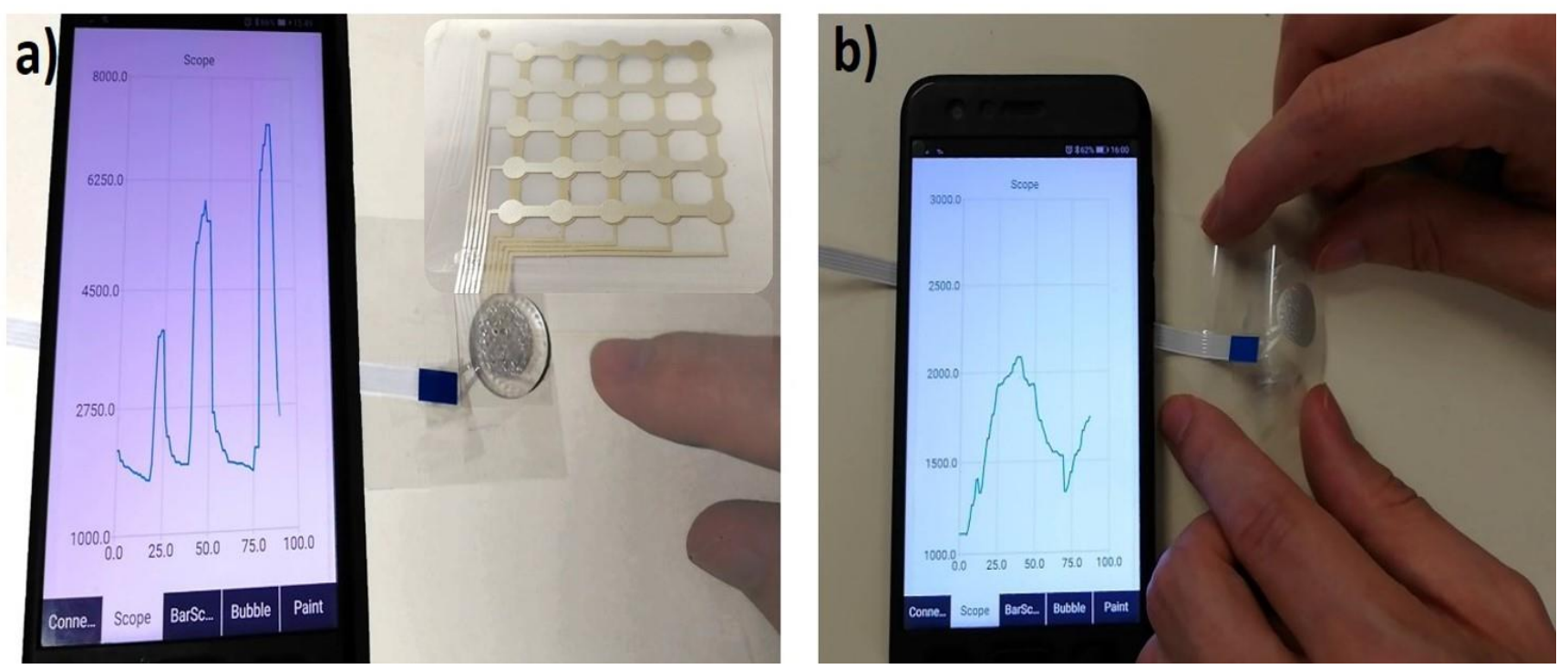



Figure 6 - Demonstration of the pressure button (a) and bending (b) sensor response. The inset in a) shows a pressure sensor matrix developed following the same processing steps. Data acquired from the sensors during manual user operation: (c) pressure, (d) bending sensors. Capacitive pressure sensor response: (e) sensitivity and linearity; (f) repeatability over 50 cycles.

Figure 6c) shows the capacitance variation of the pressure sensor when the user applies a gentle vertical compressive force is applied by finger touch. The working mechanism of the pressure sensor relies in the deformation of the dielectric layers when the material is compressed and, consequently, the capacitance increases. The same mechanism applies 
for the bending sensor presented in figure 6d). The response of the sensors (figure 6) does not show clamping effects or degradation over time, as well as adequate mechanical stability.

The data in figure 6 shows that the registered base capacitance of the sensors at rest is about $60 \mathrm{pF}$, but as predicted, the capacitance change of the pressure sensors is much higher, reaching a peak up to $300 \mathrm{pF}$ under compression and about $110 \mathrm{pF}$ under bending. A small delay is observed in the recovering time, especially in the pressure sensor, after a high peak of pressure. The same was not evident under bending, being attributed to the viscoelastic characteristics of the different layers and to the adhesion of the P(VDF-TrFE-CFE) film to the PET substrate.

Quantitative and cyclic tests were also performed on the pressure sensors in a stress testing equipment (Shimadzu model AG-IS), and the corresponding data are presented in Figure $6 e$ and $f$ ).

The sample was submitted to a compression force from 0 to $10 \mathrm{~N}$ at a test velocity of $1 \mathrm{~mm} / \mathrm{min}$ and the corresponding capacitive variation was recorded.

Figure 6e) shows that the sensitivity of the sensor is $0.03 \mathrm{kPa}^{-1}$, with a nearly linear response and no memory effect. Further, there is no need of pre-stressing the sensor, as it often happens in other type of pressure sensors such as piezoresistive ones [60]. Further, the developed sensors show higher sensibility when compared to related sensors based on CB/PDMS composites with a thin PET isolating layer [61], electrospun PVDF nanofiber membrane with carbon nanotubes [62] and single PVDF polymer [63]. The developed sensors show excellent repeatability over operating cycles, as indicated in figure $6 f$ ) for 50 cycles of compression tests.

Given the versatility and excellent response of the developed materials and considering the potential in the area of printed electronics, since the sensors can be processed both 
from solution and melt, a prototype sensor matrix was developed through the same procedure of single button, as shown in the inset of Figure 6a).

The data acquired with the pressure sensing matrix is similar to the one obtained with a single button (Figure 6). Smaller capacity electrodes required to change the series resistor with the sensor for a $10 \mathrm{M} \Omega$ one, but data acquisition followed the same steps, which grants the usability of this type of matrix.

Thus, capacitive sensors based on $\mathrm{P}(\mathrm{VDF}-\mathrm{TrFE}-\mathrm{CFE})$ terpolymer presents excellent response with high reproducibility and sensibility in pressure and bending modes, which represents an advance in materials for electronics processable by additive manufacturing technologies.

\section{Conclusions}

Samples of poly (vinylidene fluoride-trifluoroethylene-chlorofluoroethylene) (P(VDFTrFE-CFE)) were prepared by solvent casting technique to evaluate the influence of polymer concentration and processing temperature, including polymer melting conditions, on the microstructure and physicochemical properties and, consequently, on the applicability of the material in capacitive sensing. Porous and dense morphologies are obtained when the samples are processed at temperatures of $25^{\circ} \mathrm{C}$ and $210{ }^{\circ} \mathrm{C}$, respectively.

For $\mathrm{P}(\mathrm{VDF}-\mathrm{TrFE}-\mathrm{CFE})$ samples, the electroactive $\beta$ phase content and thermal degradation temperature are independent of the processing conditions. The degree of crystallinity, mechanical and dielectric and properties depend on the polymer concentration and processing temperature.

The sample with the highest dielectric constant $\left(\varepsilon^{\prime}=40\right.$ at $\left.1 \mathrm{kHz}\right)$ was obtained for the $\mathrm{P}(\mathrm{VDF}-\mathrm{TrFE}-\mathrm{CFE})$ film prepared from an initial polymer solution concentration of 15 
wt. $\%$ and processed at $210{ }^{\circ} \mathrm{C}$. This sample was used for the fabrication of capacitive pressure and bending sensors with excellent response, reproducibility, sensibility, and stability over cycling solicitation.

Thus, it is demonstrated that the obtained $\mathrm{P}(\mathrm{VDF}-\mathrm{TrFE}-\mathrm{CFE})$ films, processable from solution or from the melt, show adequate thermal, mechanical and dielectric properties for the development of pressure and deformation capacitive sensor and touchpads for a variety of applications.

\section{Acknowledgments}

The authors thank the FCT (Fundação para a Ciência e Tecnologia) for financial support under the framework of Strategic Funding grants UID/FIS/04650/2020, and UID/EEA/04436/2020; and project PTDC/FIS-MAC/28157/2017. The author also thanks the FCT for financial support under grants SFRH/BD/131729/2017 (N.P.), $\begin{array}{lllll}\text { SFRH/BD/110622/2015 (S. } & \text { G. }), & \text { SFRH/BD/140842/2018 } & \text { (J.C.B.), }\end{array}$ CEECIND/00833/2017 (R.G.) and SFRH/BPD/112547/2015 (C.M.C.). Financial support from the Spanish State Research Agency (AEI) and the European Regional Development Fund (ERFD) through the project PID2019-106099RBC43/AEI/10.13039/501100011033 and from the Basque Government Industry and Education Departments under the ELKARTEK, HAZITEK and PIBA (PIBA-2018-06) programs, respectively, are acknowledged. 


\section{References}

[1] W.H. Ko, The future of sensor and actuator systems, Sensors and Actuators A: Physical 56(1) (1996) 193-197.

[2] L.M. Swallow, J.K. Luo, E. Siores, I. Patel, D. Dodds, A piezoelectric fibre composite based energy harvesting device for potential wearable applications, Smart Materials and Structures 17(2) (2008) 025017.

[3] V.F. Cardoso, D.M. Correia, C. Ribeiro, M.M. Fernandes, S. Lanceros-Méndez, Fluorinated Polymers as Smart Materials for Advanced Biomedical Applications, Polymers 10(2) (2018) 161.

[4] W.-S. Jung, M.-J. Lee, M.-G. Kang, H.G. Moon, S.-J. Yoon, S.-H. Baek, C.-Y. Kang, Powerful curved piezoelectric generator for wearable applications, Nano Energy 13 (2015) 174-181.

[5] R.E. Newnham, G.R. Ruschau, Electromechanical Properties of Smart Materials, Journal of Intelligent Material Systems and Structures 4(3) (1993) 289-294.

[6] I.C.L. Ng, S.Y.L. Wakenshaw, The Internet-of-Things: Review and research directions, International Journal of Research in Marketing 34(1) (2017) 3-21.

[7] F. Shrouf, J. Ordieres, G. Miragliotta, Smart factories in Industry 4.0: A review of the concept and of energy management approached in production based on the Internet of Things paradigm, 2014 IEEE International Conference on Industrial Engineering and Engineering Management, 2014, pp. 697-701.

[8] S.B. Choi, Y.M. Han, Piezoelectric Actuators: Control Applications of Smart Materials, CRC Press2016.

[9] I. Giorgio, L. Galantucci, A. Della Corte, D. Del Vescovo, Piezo-electromechanical smart materials with distributed arrays of piezoelectric transducers: Current and upcoming applications, International Journal of Applied Electromagnetics and Mechanics 47 (2015) 1051-1084.

[10] C. Ribeiro, C.M. Costa, D.M. Correia, J. Nunes-Pereira, J. Oliveira, P. Martins, R. Gonçalves, V.F. Cardoso, S. Lanceros-Méndez, Electroactive poly(vinylidene fluoride)based structures for advanced applications, Nature Protocols 13(4) (2018) 681-704.

[11] T.D. Usher, K.R. Cousins, R. Zhang, S. Ducharme, The promise of piezoelectric polymers, Polymer International 67(7) (2018) 790-798. 
[12] K.S. Ramadan, D. Sameoto, S. Evoy, A review of piezoelectric polymers as functional materials for electromechanical transducers, Smart Materials and Structures 23(3) (2014) 033001.

[13] J. Oliveira, V. Correia, H. Castro, P. Martins, S. Lanceros-Mendez, Polymer-based smart materials by printing technologies: Improving application and integration, Additive Manufacturing 21 (2018) 269-283.

[14] S. Bauer, S. Bauer-Gogonea, Piezoelectric and Electrostrictive Polymers as EAPs: Devices and Applications, in: F. Carpi (Ed.), Electromechanically Active Polymers: A Concise Reference, Springer International Publishing, Cham, 2016, pp. 533-547.

[15] S. Bauer, F. Bauer, Piezoelectric Polymers and Their Applications, Piezoelectricity: Evolution and Future of a Technology, Springer Berlin Heidelberg, Berlin, Heidelberg, 2008, pp. 157-177.

[16] J.-H. Bae, S.-H. Chang, PVDF-based ferroelectric polymers and dielectric elastomers for sensor and actuator applications: a review, Functional Composites and Structures 1(1) (2019) 012003.

[17] A.J. Lovinger, Crystallization and morphology of melt-solidified poly(vinylidene fluoride), Journal of Polymer Science: Polymer Physics Edition 18(4) (1980) 793-809.

[18] H.S. Nalwa, Ferroelectric Polymers: Chemistry: Physics, and Applications, Taylor \& Francis1995.

[19] P. Martins, A.C. Lopes, S. Lanceros-Mendez, Electroactive phases of poly(vinylidene fluoride): Determination, processing and applications, Progress in Polymer Science 39(4) (2014) 683-706.

[20] M. Li, I. Katsouras, C. Piliego, G. Glasser, I. Lieberwirth, P.W.M. Blom, D.M. de Leeuw, Controlling the microstructure of poly(vinylidene-fluoride) (PVDF) thin films for microelectronics, Journal of Materials Chemistry C 1(46) (2013) 7695-7702.

[21] S. Gee, B. Johnson, A.L. Smith, Optimizing electrospinning parameters for piezoelectric PVDF nanofiber membranes, Journal of Membrane Science 563 (2018) 804-812.

[22] F. Sadeghi, S.H. Tabatabaei, A. Ajji, P.J. Carreau, Effect of PVDF characteristics on extruded film morphology and porous membranes feasibility by stretching, Journal of Polymer Science Part B: Polymer Physics 47(12) (2009) 1219-1229.

[23] C. Lee, J.A. Tarbutton, Polyvinylidene fluoride (PVDF) direct printing for sensors and actuators, The International Journal of Advanced Manufacturing Technology 104(5) (2019) 3155-3162. 
[24] M.P. Silva, C.M. Costa, V. Sencadas, A.J. Paleo, S. Lanceros-Méndez, Degradation of the dielectric and piezoelectric response of $\beta$-poly(vinylidene fluoride) after temperature annealing, Journal of Polymer Research 18(6) (2011) 1451-1457.

[25] J. Gomes, J. Serrado Nunes, V. Sencadas, S. Lanceros-Mendez, Influence of the $\beta$ phase content and degree of crystallinity on the piezo- and ferroelectric properties of poly(vinylidene fluoride), Smart Materials and Structures 19(6) (2010) 065010.

[26] C.M. Costa, R. Sabater i Serra, A. Andrio Balado, J.L. Gómez Ribelles, S. LancerosMéndez, Dielectric relaxation dynamics in poly(vinylidene fluoride)/Pb(Zr0·53Ti0.47)O3 composites, Polymer 204 (2020) 122811.

[27] V. Sencadas, S. Lanceros-Méndez, R. Sabater i Serra, A. Andrio Balado, J.L. Gómez Ribelles, Relaxation dynamics of poly(vinylidene fluoride) studied by dynamical mechanical measurements and dielectric spectroscopy, The European Physical Journal E 35(5) (2012) 41.

[28] N. Jia, Q. He, J. Sun, G. Xia, R. Song, Crystallization behavior and electroactive properties of PVDF, P(VDF-TrFE) and their blend films, Polymer Testing 57 (2017) 302306.

[29] Z. Yin, B. Tian, Q. Zhu, C. Duan, Characterization and Application of PVDF and Its Copolymer Films Prepared by Spin-Coating and Langmuir-Blodgett Method, Polymers 11(12) (2019) 2033.

[30] A. California, V.F. Cardoso, C.M. Costa, V. Sencadas, G. Botelho, J.L. GómezRibelles, S. Lanceros-Mendez, Tailoring porous structure of ferroelectric poly(vinylidene fluoride-trifluoroethylene) by controlling solvent/polymer ratio and solvent evaporation rate, European Polymer Journal 47(12) (2011) 2442-2450.

[31] H.-B. Jung, J.-W. Kim, J.-H. Lim, D.-K. Kwon, D.H. Choi, D.-Y. Jeong, Energy Storage Properties of Blended Polymer Films with Normal Ferroelectric P(VDF-HFP) and Relaxor Ferroelectric P(VDF-TrFE-CFE), Electronic Materials Letters 16(1) (2020) 47-54.

[32] N. Tsutsumi, K. Okumachi, K. Kinashi, W. Sakai, Re-evaluation of the origin of relaxor ferroelectricity in vinylidene fluoride terpolymers: An approach using switching current measurements, Scientific Reports 7(1) (2017) 15871.

[33] F. Bauer, E. Fousson, Q.M. Zhang, Recent advances in highly electrostrictive $\mathrm{P}(\mathrm{VDF}-\mathrm{TrFE}-\mathrm{CFE})$ terpolymers, IEEE Transactions on Dielectrics and Electrical Insulation 13(5) (2006) 1149-1154. 
[34] Y. Chen, X. Chen, D. Zhou, Q.-D. Shen, W. Hu, Low-temperature crystallization of P(VDF-TrFE-CFE) studied by Flash DSC, Polymer 84 (2016) 319-327.

[35] H.-M. Bao, J.-F. Song, J. Zhang, Q.-D. Shen, C.-Z. Yang, Q.M. Zhang, Phase Transitions and Ferroelectric Relaxor Behavior in $\mathrm{P}(\mathrm{VDF}-\mathrm{TrFE}-\mathrm{CFE})$ Terpolymers, Macromolecules 40(7) (2007) 2371-2379.

[36] J. Qian, J. Jiang, Y. Shen, Enhanced electrocaloric strength in P(VDF-TrFE-CFE) by decreasing the crystalline size, Journal of Materiomics 5(3) (2019) 357-362.

[37] X. Chen, L. Liu, S.-Z. Liu, Y.-S. Cui, X.-Z. Chen, H.-X. Ge, Q.-D. Shen, P(VDFTrFE-CFE) terpolymer thin-film for high performance nonvolatile memory, Applied Physics Letters 102(6) (2013) 063103.

[38] J. Shklovsky, L. Engel, Y. Sverdlov, Y. Shacham-Diamand, S. Krylov, Nanoimprinting lithography of $\mathrm{P}(\mathrm{VDF}-\mathrm{TrFE}-\mathrm{CFE})$ for flexible freestanding MEMS devices, Microelectronic Engineering 100 (2012) 41-46.

[39] A. Javadi, Y. Xiao, W. Xu, S. Gong, Chemically modified graphene/P(VDF-TrFECFE) electroactive polymer nanocomposites with superior electromechanical performance, Journal of Materials Chemistry 22(3) (2012) 830-834.

[40] D.M. Correia, R. Gonçalves, C. Ribeiro, V. Sencadas, G. Botelho, J.L.G. Ribelles, S. Lanceros-Méndez, Electrosprayed poly(vinylidene fluoride) microparticles for tissue engineering applications, RSC Advances 4(62) (2014) 33013-33021.

[41] A. Schönhals, F. Kremer, Theory of Dielectric Relaxation, in: F. Kremer, A. Schönhals (Eds.), Broadband Dielectric Spectroscopy, Springer Berlin Heidelberg, Berlin, Heidelberg, 2003, pp. 1-33.

[42] J.S. Nunes, N. Castro, S. Gonçalves, N. Pereira, V. Correia, S. Lanceros-Mendez, Marked Object Recognition Multitouch Screen Printed Touchpad for Interactive Applications, Sensors 17(12) (2017) 2786.

[43] C. Mendes-Felipe, J.C. Barbosa, S. Gonçalves, N. Pereira, C.M. Costa, J.L. VilasVilela, S. Lanceros-Mendez, High dielectric constant UV curable polyurethane acrylate/indium tin oxide composites for capacitive sensing, Composites Science and Technology 199 (2020) 108363.

[44] A. Garinei, R. Marsili, Development of a new capacitive matrix for a steering wheel's pressure distribution measurement, International Journal of Industrial Ergonomics 44(1) (2014) 114-119.

[45] J.C.C. Ferreira, T.S. Monteiro, A.C. Lopes, C.M. Costa, M.M. Silva, A.V. Machado, S. Lanceros-Mendez, Variation of the physicochemical and morphological characteristics 
of solvent casted poly(vinylidene fluoride) along its binary phase diagram with dimethylformamide, Journal of Non-Crystalline Solids 412 (2015) 16-23.

[46] R.E. Sousa, J.C.C. Ferreira, C.M. Costa, A.V. Machado, M.M. Silva, S. LancerosMendez, Tailoring poly(vinylidene fluoride-co-chlorotrifluoroethylene) microstructure and physicochemical properties by exploring its binary phase diagram with dimethylformamide, Journal of Polymer Science Part B: Polymer Physics 53(11) (2015) 761-773.

[47] R. Magalhães, N. Durães, M. Silva, J. Silva, V. Sencadas, G. Botelho, J.L. Gómez Ribelles, S. Lanceros-Méndez, The Role of Solvent Evaporation in the Microstructure of Electroactive $\beta$-Poly(Vinylidene Fluoride) Membranes Obtained by Isothermal Crystallization, Soft Materials 9(1) (2010) 1-14.

[48] F. Fang, W. Yang, W. Yang, Mechanical flexible and electric fatigue resistant behavior of relaxor ferroelectric terpolymer, Journal of Applied Physics 106(3) (2009) 034106.

[49] F. Fang, W. Yang, M.Z. Zhang, Electric fatigue in poly(vinylidene fluoridetrifluoroethylene) 68/32 copolymer films under cyclic electric field, Journal of Applied Physics 101(4) (2007) 044902.

[50] V. Sencadas, R. Gregorio, S. Lanceros-Méndez, $\alpha$ to $\beta$ Phase Transformation and Microestructural Changes of PVDF Films Induced by Uniaxial Stretch, Journal of Macromolecular Science, Part B 48(3) (2009) 514-525.

[51] J. Nunes-Pereira, P. Martins, V.F. Cardoso, C.M. Costa, S. Lanceros-Méndez, A green solvent strategy for the development of piezoelectric poly(vinylidene fluoridetrifluoroethylene) films for sensors and actuators applications, Materials \& Design 104 (2016) 183-189.

[52] O.N.L. Smith, Y. Kim, M. Kathaperumal, M.R. Gadinski, M.-J. Pan, Q. Wang, J.W. Perry, Enhanced Permittivity and Energy Density in Neat Poly(vinylidene fluoridetrifluoroethylene-chlorotrifluoroethylene) Terpolymer Films through Control of Morphology, ACS Applied Materials \& Interfaces 6(12) (2014) 9584-9589.

[53] Y. Wang, S. Lu, M. Lanagan, Q. Zhang, Dielectric relaxation of relaxor ferroelectric $\mathrm{P}(\mathrm{VDF}-\mathrm{TrFE}-\mathrm{CFE})$ terpolymer over broad frequency range, IEEE Transactions on Ultrasonics, Ferroelectrics, and Frequency Control 56(3) (2009) 444-449.

[54] J.-F. Capsal, E. Dantras, C. Lacabanne, Molecular mobility interpretation of the dielectric relaxor behavior in fluorinated copolymers and terpolymers, Journal of NonCrystalline Solids 363 (2013) 20-25. 
[55] G. Botelho, S. Lanceros-Mendez, A.M. Gonçalves, V. Sencadas, J.G. Rocha, Relationship between processing conditions, defects and thermal degradation of poly(vinylidene fluoride) in the $\beta$-phase, Journal of Non-Crystalline Solids 354(1) (2008) $72-78$.

[56] D.M. Correia, C.M. Costa, J. Nunes-Pereira, M.M. Silva, G. Botelho, J.L.G. Ribelles, S. Lanceros-Méndez, Physicochemical properties of poly(vinylidene fluoridetrifluoroethylene)/poly(ethylene oxide) blend membranes for lithium ion battery applications: Influence of poly(ethylene oxide) molecular weight, Solid State Ionics 268 (2014) 54-67.

[57] M.M. Maciel, S. Ribeiro, C. Ribeiro, A. Francesko, A. Maceiras, J.L. Vilas, S. Lanceros-Méndez, Relation between fiber orientation and mechanical properties of nanoengineered poly(vinylidene fluoride) electrospun composite fiber mats, Composites Part B: Engineering 139 (2018) 146-154.

[58] B. Chu, X. Zhou, K. Ren, B. Neese, M. Lin, Q. Wang, F. Bauer, Q.M. Zhang, A Dielectric Polymer with High Electric Energy Density and Fast Discharge Speed, Science 313(5785) (2006) 334-336.

[59] H. Arisawa, O. Yano, Y. Wada, Dielectric loss of poly(vinylidene fluoride) at low temperatures and effect of poling on the low temperature loss, Ferroelectrics 32(1) (1981) $39-41$.

[60] P. Costa, S. Gonçalves, H. Mora, S.A.C. Carabineiro, J.C. Viana, S. LancerosMendez, Highly Sensitive Piezoresistive Graphene-Based Stretchable Composites for Sensing Applications, ACS Applied Materials \& Interfaces 11(49) (2019) 46286-46295. [61] M. Pruvost, W.J. Smit, C. Monteux, P. Poulin, A. Colin, Polymeric foams for flexible and highly sensitive low-pressure capacitive sensors, npj Flexible Electronics 3(1) (2019) 7.

[62] X. Yang, Y. Wang, X. Qing, A flexible capacitive sensor based on the electrospun PVDF nanofiber membrane with carbon nanotubes, Sensors and Actuators A: Physical 299 (2019) 111579.

[63] K. Arshak, D. Morris, A. Arshak, O. Korostynska, K. Kaneswaran, Investigation into the pressure sensing properties of PVDF and PVB thick film capacitors, 2006 29th International Spring Seminar on Electronics Technology, 2006, pp. 334-339. 